\title{
Table of national and international legislation
}

\section{United Kingdom}

Patent and Designs Act 1907

s. $75 \mathbf{3 0 8}$

Patents Act 1949
s. $32(1)(d) \quad 307$
s. 101

Patents Act 1977

s. 2(6) $\mathbf{3 4 5}-\mathbf{3 4 7}, \mathbf{3 5 3}, \mathbf{3 5 7}, \mathbf{3 5 8}$, 362,372

s. 4(2) $\mathbf{3 3 4}-\mathbf{3 3 8}, \mathbf{3 4 2}, \mathbf{3 4 5}, \mathbf{3 4 7}$, $351,353,358,359$

s. $4(3) \quad 345, \mathbf{3 4 7}, 358$

s. $4 \mathrm{~A}(2) \quad \mathbf{3 4 5}, \mathbf{3 5 8}$

s. $4 \mathrm{~A}(3) \quad 355$

s. $4 \mathrm{~A}(4) \quad 356$

s. $36 \quad \mathbf{3 8}$

s. $37 \quad 38$

s. $48(1)(a) \quad 54$

s. $48(1)(b) \quad 55$

s. $48(2)(a) \quad 55$

s. $48(1)(c) \quad 55$

s. $48(2)(a) \quad 55$

s. $48(3)$ (a) $\mathbf{5 5}$

s. $48(3)(d) \quad \mathbf{5 5}$

s. $48(3)(\mathrm{e}) \mathbf{5 5}$

s. $60 \mathbf{6 5}, \mathbf{6 8}, \mathbf{3 0 7}$

s. $60(5) \quad 13$

s. $60(5)$ (a) $\mathbf{6 8}$

s. $60(5)(b) 13,68$

s. $72(1) \quad 307$

s. 91(1) 348

s. $130(7) \quad 332,333$
Statute of Monopolies

s. $67,287,289,296,298,299$, 301, 304, 305, 311, 313, 314, $322,330,333,434$

European Patent Convention

Art. 4(3) 96

Art. 52(1) 95, 96, 101-103,

191, 202-204, 241, 420

Art. 52(2)(a) 203

Art. 52(4) 1973 2, 71, 87, 227, 252

Art. 52(4) 71, 72, 76-82, 86, 88-91, 93-98, 101, 102, 104-106, 108-110, 114, 117, 122-125, 127,-129, 131-

$141,144,149-155,157-164$, 166, 170-174, 177-179, 185, 186, 189, 190, 192-194, 196-201, 203-208, 210, 212, 215-217, 228, 235-240, 242, 249-252, 256-258, 261-264, 266, 267, 280, 285, 286, 325, $327,328,335,343,354,433$

Art.53(a) 8, 113, 115, 177, 223-227, 314, 438, 440

Art. 53(c) 2, 71 86-88, 95, 102, $103,106,113,114,115,117$, $122,126,134,140,141,143-$ $146,155,161,162,167,168$, 176-178, 180-184, 192, 202, 219, 220, 229, 255, 259, 261, 269-275, 277, 280, 281, 282, $285,343,354,372,385,433$, 437-440 
Art. 54(4) 6, 229, 240, 253, 254, 259, 260, 268, 271-274, 276-278, 281-284, 441 Art. 54(5) 1973 6, 115, 229, 230, 234, 235, 237, 239, 240,247, 251-254, 256-262, 268, 273, 274, 276, 278, 280, 281, 283, 284, 286, 327, 328, $345,346,350,354,372,441$ Art. 54(5) 6, 115, 185, 229, 240, 253-255, 259-261, 268-286, 347, 356, 372, 441, 442

\section{United States}

Patent Law 35 U.S.C.

S. 101 374, 392-395, 403, 404, 407, 409-411, 413,415-418, $420,422-426,431,432,435$

s. 103 (b) $\mathbf{3 8 6}$

s. 271(a) $\mathbf{3 9 0}$

s. 271(b) $\mathbf{3 9 8}$

Medical Procedures and Affordability Act

S. $287(\mathrm{c}) \quad \mathbf{3 8 4}, \mathbf{3 8 5}$

s. $287(\mathrm{c})(1) \quad \mathbf{3 8 4}, \mathbf{3 8 6}$

s. $287(\mathrm{c})(2) \quad \mathbf{3 8 4}, \mathbf{3 8 6}, 391$

s. $287(\mathrm{c})(2)(\mathrm{A}) \quad \mathbf{3 8 4}$

s. $287(\mathrm{c})(2)(\mathrm{B}) \quad \mathbf{3 8 4}$

s. $287($ c)(2)(b)(ii) 389

s. $287(\mathrm{c})(3) \quad 291$

s. $287(\mathrm{c})(3)(\mathrm{A}) \quad \mathbf{3 8 5}, \mathbf{3 8 6}$

s. $287($ c)(3)(A)(i) 387

s. $287(\mathrm{c})(3)(\mathrm{B}) \mathbf{3 8 6}$

s. $287(\mathrm{c})(3)(\mathrm{B})(\mathrm{i}) \quad \mathbf{3 8 4}, \mathbf{3 8 6}$

s. 287 (c)(3)(B)(ii) $\mathbf{3 8 4 ,}, \mathbf{3 8 6}, 387$

s. 287 (c)(3)(B)(iii) $\mathbf{3 8 4 ,} 386,387$

s. $287(\mathrm{c})(3)(\mathrm{C})(\mathrm{i}) \quad \mathbf{3 8 5}, \mathbf{3 8 6}$

s. $287(\mathrm{c})(3)(\mathrm{C})(\mathrm{ii}) \quad \mathbf{3 8 5}, \mathbf{3 8 6}$

s. $287(\mathrm{c})(3)(\mathrm{D}) \quad \mathbf{3 8 5}, \mathbf{3 8 8}, \mathbf{3 8 9}$, 390

s. $287(\mathrm{c})(3)(\mathrm{E}) \quad \mathbf{3 8 5}, 391$

s. $287(\mathrm{c})(3)(\mathrm{F})(\mathrm{i}) \quad \mathbf{3 8 6}$

s. $287(\mathrm{c})(3)(\mathrm{F})(\mathrm{ii}) \quad \mathbf{3 8 6}$

s. $287(\mathrm{c})(4) \quad \mathbf{3 8 5}, 391$
Genomic Research and Diagnostic Accessibility Act

s. 2(1) 391

s. 3(a)(1) 391

s. 3(a)(3) 391

s. 3(b) 391

s. 3(c) 392

\section{European Union}

Biotech Directive 1998

$\begin{array}{ll}\text { R. } 16 & \mathbf{1 1 5} \\ \text { R. } 20 & \mathbf{1 1 5} \\ \text { R. } 21 & \mathbf{1 5 5} \\ \text { R. } 22 & \mathbf{1 1 5} \\ \text { R. } 23 & \mathbf{1 1 5} \\ \text { R. } 24 & \mathbf{1 1 5} \\ \text { R. } 26 & \mathbf{2 2 4} \\ \text { R. } 35 & \mathbf{1 2 2}, \mathbf{2 2 1} \\ \text { R. } 36 & \mathbf{2 2 2} \\ \text { R. } 37 & \mathbf{2 2 2} \\ \text { R. } 38 & \mathbf{2 2 2} \\ \text { R. 39 } & \mathbf{2 2 2} \\ \text { R. } 40 & \mathbf{2 2 2} \\ \text { R. } 41 & \mathbf{1 1 5} \\ \text { R. } 42 & \mathbf{2 2 1} \\ \text { R. } 45 & \mathbf{2 2 1} \\ \text { Art. 5(1) } & \mathbf{2 2 2} \\ \text { Art. 5(2) } & \mathbf{2 2 2} \\ \text { Art. 5(3) } & \mathbf{2 6 , 2 2 2} \\ \text { Art. 6(1) } & \mathbf{2 2 2} \\ \text { Art. 6(2) } & \mathbf{2 2 2}, \mathbf{2 2 3} \\ \text { Art. 9 } & \mathbf{2 2 2}\end{array}$

\section{International}

TRIPs Agreement

Art. 27(3) 47

\section{Other}

AMA's Code of Medical Ethics, E-9.08 New Medical

Procedures $\mathbf{6 2}$

Declaration on the TRIPs Agreements and Public Health 47 
xxiv Medical patent law - the challenges of medical treatment

International Code of Medical Ethics 62, 67

World Association International

Code of Medical Ethics $\mathbf{6 2}$ 